ARTIGO ORIGINAL

OriginAl ARTICLE

\title{
Qualidade de Vida em Pacientes com Osteoporose: Correlação entre OPAQ e SF-36
}

\section{Quality of Life in Patients with Osteoporosis: Correlation between OPAQ and SF-36}

\author{
Maria Carolina Davel Lemos ${ }^{(1)}$, Samira Tatiyama Miyamoto(2), Valéria Valim ${ }^{(3)}$, Jamil Natour ${ }^{(4)}$
}

\section{RESUMO}

Objetivo: correlacionar o instrumento de avaliação de qualidade de vida específico para osteoporose, o Osteoporosis Assessment Questionnaire (OPAQ), com o instrumento genérico, o The Medical Outcomes Study 36 - Item Short Form Health Survery (SF-36). Pacientes e Métodos: estudo observacional de corte transversal que incluiu 40 mulheres acima de 60 anos, diagnosticadas com osteoporose. Houve um momento para avaliação e um único examinador. Para a análise estatística foram utilizados coeficiente correlação de Pearson e análise fatorial do OPAQ. Resultados: houve correlação entre os domínios e componentes finais do SF-36 com os domínios do OPAQ. Evidenciou-se que os coeficientes mais altos foram entre o OPAQ Sintomas e os seguintes domínios do SF-36: dor $(\mathrm{r}=-0,6)$, aspectos sociais $(\mathrm{r}=-0,6 \%)$ e saúde mental $(\mathrm{r}=-0,5)$. Quase todos os aspectos do SF-36 se correlacionaram com o OPAQ Sintomas. E, ainda, por análise fatorial do OPAQ, observa-se que os seus 18 componentes representaram $63 \%$ de carga fatorial, ou seja, eles explicaram o valor total de impacto da osteoporose na qualidade de vida e agruparam-se em cinco fatores: fator 1 (mobilidade, cuidados próprios, independência e trabalho doméstico), fator 2 (flexibilidade, movimentação e sono), fator 3 (dor nas costas, dor relacionada à osteoporose, fadiga e humor), fator 4 (medo de cair, atividades sociais, apoio familiar e tensão) e fator 5 (andarinclinar, imagem corporal e trabalho). Conclusão: foi encontrada a correlação esperada entre SF-36 e OPAQ, que é um instrumento de avaliação específico com capacidade para avaliar aspectos específicos e gerais da qualidade de vida nesta população.

Palavras-chave: qualidade de vida, osteoporose, SF-36, OPAQ.

\begin{abstract}
Objective: to correlate the quality of life assessment instrument, specific to osteoporosis, OPAQ (Osteoporosis Assessment Questionnaire), with the generic instrument SF-36 (Item Short Form Health Survey). Patients and Methods: the cross sectional observational study included 40 female patients older than 60 years with osteoporosis. A single assessment was always conducted by the same evaluator. For the statistics analisys the Pearson's coefficient and the factor analysis to OPAQ were used. Results: there was a correlation between the domains and final components of SF-36 with the domains of OPAQ. The higher coefficients were between the OPAQ Symptoms and some domains of SF-36: pain $(r=-0,6)$, social aspects $(r=-0,6)$ and mental health $(r=-0,5)$. Almost every aspect of SF-36 was correlated with the OPAQ Symptoms. By factor analysis of OPAQ it was observed that the 18 components of OPAQ represented $63 \%$ of factor load, and they explained the total account of osteoporosis's impact in the quality of life and were grouped in five factors: factor 1 (mobility, self-care, independence and household tasks), factor 2 (flexibility, transfers and sleep), factor 3 (backache, pain related to osteoporosis, fatigue and mood), factor 4 (fear of fall, social activity, family support and tension) and factor 5 (walking and bending, body image and work). Conclusion: the expected correlation was achieved between SF-36 and OPAQ, wich is a specific assessment instrument capable of assessing particular and general aspects of the quality of life in patients with osteoporosis.
\end{abstract}

Keywords: Quality of life, osteoporosis, SF-36, OPAQ.

Divisão de Reumatologia da Universidade Federal de São Paulo (UNIFESP). Recebido em 03/1 1/05. Aprovado, após revisão, em 28/08/06.

1. Fisioterapeuta, Especialista em Reumatologia.

2. Fisioterapeuta, Mestre em Ciências.

3. Médica, Doutora em Reumatologia, participante do PRO-NUCLEAR.

4. Reumatologista livre docente, Chefe do Serviço de Reabilitação e do Setor de Coluna Vertebral da UNIFESP.

Endereço para correspondência: Jamil Natour, Divisão de Reumatologia da UNIFESP, Rua Botucatu, 740, Vila Clementino, CEP 04023-900, São Paulo, SP, Brasil, e-mail: jnatour@reumato.epm.br 


\section{INTRODUÇÃO}

A osteoporose é uma doença caracterizada por diminuição da massa óssea e deterioração da microarquitetura do tecido ósseo, levando a um aumento na fragilidade dos ossos e, conseqüentemente, aumento no risco de fraturas ${ }^{(1)}$.

Cada vez mais, a osteoporose é reconhecida como enfermidade limitante da qualidade de vida. A perda de independência, decorrente da incapacidade de deambular, é a principal conseqüência da fratura de quadril, seja por limitação funcional ou por medo de quedas. Essa inatividade leva à piora da osteoporose e aumenta ainda mais os riscos de quedas e novas fraturas ${ }^{(2)}$.

Em relação ao estudo da qualidade de vida na osteoporose, poucos trabalhos na literatura internacional foram realizados e publicados. Tais trabalhos mostram que a qualidade de vida, principalmente o aspecto social, é influenciada pela ocorrência das fraturas ${ }^{(3,4,5,6,7)}$.

A fim de avaliar a qualidade de vida e o estado global de saúde no acompanhamento dos pacientes de diferentes doenças, foram desenvolvidos questionários genéricos e específicos $^{(8,9,10)}$. Na literatura, os questionários já utilizados em pacientes com osteoporose são: Osteoporosis-Targeted

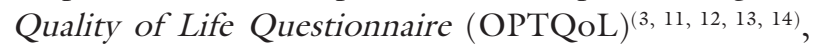
Osteoporosis Assessment Questionnaire (OPAQ) $)^{(13,15,16,17)}$, Osteoporosis Quality-of-Life Questionnaire(OQLQ) $)^{(13,18,19)}$, Quality-of-Life Questionnaire of the European Foundation for Osteoporosis (QUALEFFO) ${ }^{(13,18,20)}$, Osteoporosis Functional Disability Questionnaire (OFDQ) ${ }^{(13)}$, Qualityof-Life Questionnaire in Osteoporosis (QUALIOST) ${ }^{(13,21)}$, The Medical Outcomes Study 36 - Item Short Form Health Survery (SF-36) $7,11,12,13,18,21)$, Facts on Osteoporosis Quiz $(\text { FOOQ })^{(22)}$, Beck Depression Inventory (BDI $)^{(23)}$.

O OPAQ é o único questionário específico para osteoporose traduzido e adaptado para o português e, por isso, é de grande utilidade na prática clínica e em pesquisas ${ }^{(17)}$. O OPAQ possui 5 questões gerais sobre saúde e qualidade de vida na parte A e outros 18 componentes independentes na parte $\mathrm{B}$ reunidos em 4 domínios que avaliam função física, estado psicológico, sintomas e interação social ${ }^{(15)}$. O OPAQ é utilizado com mais freqüência em outros países além do Brasil, como os Estados Unidos e Austrália ${ }^{(13,16)}$.

O The Medical Outcomes Study 36-Item Short Form Health Survery (SF-36) é um instrumento de avaliação genérico de qualidade de vida validado, de fácil administração e compreensão. É um questionário multidimensional, formado por 36 itens, englobados em 8 componentes ou domínios: capacidade funcional, aspectos físicos, dor, estado geral da saúde, vitalidade, aspectos sociais, aspectos emocionais e saúde mental e pode ser sintetizado em dois componentes, mental e físico. Apresenta um escore final de 0 a 100, no qual zero corresponde ao pior estado geral e 100 ao melhor estado de saúde ${ }^{(24,25,26)}$. O SF-36 é o questionário genérico mais utilizado na osteoporose $\mathrm{e}^{(7,11,12,18,21,27)}$. A versão brasileira do SF-36 é muito utilizada em várias áreas da saúde ${ }^{(26)}$.

Nosso objetivo foi comparar o OPAQ, um instrumento específico, ao SF-36, um instrumento genérico, bem como estudar a inter-relação entre seus domínios, testando sua capacidade específica de medir a qualidade de vida dos pacientes com osteoporose comparando com um instrumento genérico já conhecido (SF-36).

\section{PACIENTES E MÉTODOS}

O estudo é do tipo observacional de corte transversal e incluiu 40 pacientes do sexo feminino com idade acima de 60 anos e diagnóstico de osteoporose (com ou sem fratura $)^{(28)}$. O grupo de pacientes teve sua qualidade de vida avaliada por meio dos instrumentos de avaliação OPAQ e do SF-36, aplicados por um único avaliador no mesmo dia, ficando desprovidos de qualquer outra intervenção. Os dados pessoais coletados foram catalogados em uma ficha de avaliação individual. Os participantes avaliados não tiveram nenhuma instrução sobre prevenção ou tratamento previamente pelo estudo.

As pacientes foram recrutadas na clínica escola da EMESCAM (Escola Superior de Ciências da Santa Casa de Misericórdia de Vitória/ES). Todas as pacientes preencheram um termo de consentimento livre e esclarecido para participarem do estudo.

A análise estatística utilizada nesse estudo constou de análise descritiva para os dados populacionais, coeficiente de correlação de Pearson e análise fatorial. Utilizamos o programa estatístico SPSS 8.0.

O coeficiente de correlação de Pearson foi calculado para verificar se há correlação entre os domínios e componentes finais do SF-36 e os domínios do OPAQ, com p< 0,01 . A estrutura fatorial dos 18 itens do OPAQ foi avaliada a partir de extração dos componentes principais com rotação Varimax, sendo feita para a obtenção da porcentagem de variância de explicação do modelo e para a inter-relação dos seus domínios. A análise fatorial agrupa um conjunto de técnicas estatísticas com o objetivo de determinar a interdependência entre variáveis e promover sua redução. É aplicada, especificamente, à validação de instrumentos, 
visando relacionar os conceitos teóricos subjacentes às medidas escolhidas para representar estes conceitos.

\section{RESULTADOS}

Todas as questões do SF-36 e do OPAQ foram respondidas. Apenas cinco pacientes responderam às questões sobre atividade sexual, pois as demais não praticavam sexo. O tempo médio de aplicação do SF-36 foi de $20 \mathrm{minu}$ tos e do OPAQ foi de 40 minutos. Os valores dos dados populacionais, como idade, peso, altura e índice de massa corpórea (IMC) da amostra, estão na Tabela 1 .

O questionário SF-36 teve seus domínios calculados separadamente e agrupados nos componentes físico e mental. Os valores médios do SF-36 estão apresentados na Tabela 2 e os do OPAQ estão na Tabela 3.

TABELA 1

VALORES DAS MÉdias E DESVIOS-PADRÃo DA IDADE, PESO, ALTURA E MEDIDA DE MASSA CORPÓREA

\begin{tabular}{cc}
\hline Dados Populacionais & Média (DP) \\
\hline Idade $(\mathrm{anos})$ & $72,17(6,48)$ \\
Peso $(\mathrm{kg})$ & $58,96(9,31)$ \\
Altura $(\mathrm{m})$ & $1,55(0,06)$ \\
IMC $\left(\mathrm{kg} / \mathrm{m}^{2}\right)$ & $24,28(3,49)$ \\
\hline
\end{tabular}

IMC: índice de massa corpórea; DP: desvio-padrão.

TABELA 2

VALORES DOS OITO DOMÍNIOS E DOS DOIS COMPONENTES FINAIS (FÍSICO E MENTAL) DO SF-36

\begin{tabular}{lccc}
\hline $\begin{array}{l}\text { Domínios e } \\
\text { Componentes }\end{array}$ & Média (DP) & Mínimo & Máximo \\
\hline Capacidade funcional & $55,75(16,46)$ & 20 & 85 \\
\hline Aspectos físicos & $45,62(34,84)$ & 0 & 100 \\
\hline Dor & $57,37(17,83)$ & 22 & 100 \\
\hline Estado geral de saúde & $65,92(20,99)$ & 10 & 92 \\
\hline Vitalidade & $57,75(16,94)$ & 20 & 85 \\
\hline Aspectos sociais & $71,56(19,81)$ & 25 & 100 \\
\hline Aspectos emocionais & $45,81(38,25)$ & 0 & 100 \\
\hline Saúde mental & $65(20,75)$ & 8 & 92 \\
\hline CF - final & $37,66(7,28)$ & 14,53758 & 54,88466 \\
\hline CM - final & $37,27(12,57)$ & 13,53779 & 61,93693 \\
\hline
\end{tabular}

CF-final: componente físico final; CM-final: componente mental final.

Rev Bras Reumatol, v. 46, n.5, p. 323-328, set/out, 2006
TABELA 3

VALORES DOS 18 COMPONENTES E DOS 4 DOMÍNIOS DO OPAQ

\begin{tabular}{|c|c|c|c|}
\hline Componentes e Domínios & Média (DP) & Mínimo & Máximo \\
\hline c1 - mobilidade & $2,9(1,85)$ & 0 & 6 \\
\hline c2 - andar e inclinação & $4,08(2,40)$ & 0 & 9 \\
\hline c3 - dor nas costas & $4,33(2,39)$ & 0 & 9,5 \\
\hline c4 - flexibilidade & $1,35(1,69)$ & 0 & 6,25 \\
\hline c5 - cuidados próprios & $0,06(0,31)$ & 0 & 1,875 \\
\hline c6 - trabalho doméstico & $1,53(1,98)$ & 0 & 8,75 \\
\hline c7 - movimentação & $1,56(1,48)$ & 0 & 6,25 \\
\hline c8 - medo de cair & $6,01(2,57)$ & 0,5 & 10 \\
\hline c9 - atividades sociais & $3,17(1,38)$ & 1 & 6 \\
\hline c10 - apoio familiar & $0,92(1,80)$ & 0 & 8,75 \\
\hline $\begin{array}{l}\text { c11 - dor relacionada à } \\
\text { osteoporose }\end{array}$ & $3,47(2,04)$ & 0 & 8 \\
\hline c12 - sono & $3,82(2,29)$ & 0 & 8,75 \\
\hline c13 - fadiga & $3,31(2,33)$ & 0 & 10 \\
\hline c14 - trabalho & $2,06(1,22)$ & 0 & 4,375 \\
\hline c15 - nível de tensão & $3,88(2,08)$ & 0,5 & 10 \\
\hline c16 - humor & $2,08(1,66)$ & 0 & 7,5 \\
\hline c17 - imagem corporal & $4,34(2,23)$ & 0 & 8,75 \\
\hline c18 - independência & $3,16(2,03)$ & 0 & 6,66 \\
\hline Domínio físico & $13,56(6,86)$ & 0 & 38,75 \\
\hline Domínio psicológico & $19,49(5,21)$ & 10,29 & 34,583 \\
\hline Domínio sintomas & $14,95(6,54)$ & 0,625 & 31,625 \\
\hline Domínio social & $4,09(2,54)$ & 1 & 13,75 \\
\hline
\end{tabular}

A maioria das pacientes está satisfeita em relação à sua vida e saúde; $67 \%$ referem sua vida como agradável ou muito satisfatória, $28 \%$ regular e apenas $5 \%$ como muito insatisfatória. De uma maneira geral, $3 \%$ disseram que sua saúde é excelente, $13 \%$ muito boa, $57 \%$ boa e $27 \%$ regular. No entanto, parte delas tem uma impressão de deterioração da sua saúde. Comparando há um ano, 18\% disseram estar "um pouco pior", 52\% "mais ou menos". Apenas 
$30 \%$ referiram-se "um pouco melhor" ou "muito melhor" (questões C1, C3, C4 e C5 do OPAQ).

A questão $\mathrm{C} 2$ pontua de 0 a 10 a qualidade de vida com um todo ("Você poderia assinalar o número que melhor indica a nota que você daria à sua qualidade de vida como um todo?") teve média 6,85 e desvio-padrão (DP) $\pm 2,41$.

Por análise fatorial os 18 componentes do OPAQ (parte B) explicam $63 \%$ do impacto da osteoporose na qualidade de vida, através da variabilidade total explicada pelo modelo com cinco cargas fatoriais somadas (Tabela 4). Esta análise agrupou os componentes que tiveram uma boa correlação entre si nos cinco fatores a seguir.

O fator 1 ( $15 \%$ de carga fatorial) agrupou itens relativos do indivíduo com seu trabalho, como mobilidade, cuidados próprios, independência e trabalho doméstico.

O fator 2 (15\%) agrupou as variáveis que representam a dimensão de flexibilidade, movimentação e sono.

TABELA 4

ANÁlise FATORIAL DOS 18 COMPONENTES - PARTE B

DO OPAQ COM OS VALORES DE CARGAS FATORIAIS E PORCENTAGENS DE VARIÂNCIA DE CADA FATOR

\begin{tabular}{|c|c|c|c|c|c|}
\hline & Fator $1(15 \%)$ & Fator $2(15 \%)$ & Fator $3(14 \%)$ & ) Fator $4(11 \%)$ & Fator $5(8 \%)$ \\
\hline c1 & 0,713 & 0,237 & 0,035 & $-0,043$ & $-0,236$ \\
\hline c2 & 0,433 & 0,256 & $-0,021$ & $-0,278$ & 0,549 \\
\hline c3 & 0,245 & 0,065 & 0,792 & $-0,084$ & $-0,016$ \\
\hline c4 & 0,189 & 0,830 & 0,062 & 0,087 & 0,043 \\
\hline c5 & 0,672 & $-0,048$ & $-0,068$ & 0,096 & 0,311 \\
\hline c6 & 0,710 & 0,346 & 0,036 & $-0,032$ & $-0,031$ \\
\hline c7 & 0,213 & 0,790 & 0,122 & 0,154 & 0,080 \\
\hline C8 & 0,180 & 0,465 & 0,291 & $-0,626$ & 0,206 \\
\hline c9 & 0,357 & $-0,023$ & 0,196 & 0,537 & $-0,007$ \\
\hline c10 & $-0,140$ & 0,091 & 0,034 & 0,798 & 0,162 \\
\hline c11 & $-0,019$ & 0,366 & 0,784 & $-0,033$ & 0,065 \\
\hline c12 & $-0,231$ & 0,685 & 0,279 & $-0,162$ & $-0,203$ \\
\hline c13 & $-0,252$ & 0,347 & 0,446 & 0,463 & $-0,151$ \\
\hline c14 & 0,086 & 0,358 & 0,321 & $-0,216$ & $-0,601$ \\
\hline c15 & $-0,217$ & 0,247 & 0,467 & 0,506 & $-0,056$ \\
\hline c16 & $-0,043$ & $-0,017$ & 0,758 & 0,319 & $-0,021$ \\
\hline c17 & $-0,088$ & 0,050 & 0,115 & 0,006 & 0,797 \\
\hline c18 & 0,775 & $-0,159$ & 0,062 & $-0,228$ & $-0,051$ \\
\hline
\end{tabular}

c1: mobilidade; c2: andar e inclinação; c3: dor nas costas; c4: flexibilidade; c5: cuidados próprios; c6: trabalho doméstico; c7: movimentação; c8: medo de cair; c9: atividades sociais; c10: apoio familiar; c11: dor pela osteoporose; c12: sono; c13: fadiga; c14: trabalho; c15: nível de tensão; c16: humor; c17: imagem corporal; c18: independência.

Os componentes que tiveram correlação entre si em um mesmo fator estão destacados em colunas.
O fator 3 (14\%) agrupou uma dimensão para sintomas da doença, como dor nas costas, dor pela osteoporose, fadiga e humor.

O fator $4(11 \%)$ agrupou itens no contexto dos relacionamentos, portanto, medo de cair, atividades sociais, apoio familiar e tensão.

O fator 5 (8\%) agrupou as ações da vida diária, como andar/inclinação, imagem corporal e trabalho.

As mais importantes dimensões que explicam a qualidade de vida dos pacientes estão nos fatores de 1 a 3 , pois eles responderam quase $45 \%$ da variância total (63\%) vistos na Tabela 4.

$\mathrm{Na}$ Tabela 5, estão representados os coeficientes de correlação de Pearson entre os domínios e componentes finais (físico e mental) do SF-36 e os domínios físico, psicológico, sintomas e social do OPAQ, com as significâncias estatísticas.

TABELA 5

Coeficientes de correlação de Pearson (CCP) entre OS DOMÍNIOS E COMPONENTES FINAIS DO SF-36 E OS DOMÍNIOS DO OPAQ

\begin{tabular}{|c|c|c|c|c|c|}
\hline SF-36 & & $\begin{array}{l}\text { OPAQ } \\
\text { Físico }\end{array}$ & $\begin{array}{c}\text { OPAQ } \\
\text { Psicológico }\end{array}$ & $\begin{array}{c}\text { OPAQ } \\
\text { Sintomas }\end{array}$ & $\begin{array}{l}\text { OPAQ } \\
\text { Social }\end{array}$ \\
\hline \multirow[t]{2}{*}{ CF } & $\mathrm{CCP}$ & $-0,41382^{*}$ & 0,048687 & 0,00286 & 0,075518 \\
\hline & $p$ & 0,007945 & 0,765445 & 0,986026 & 0,643268 \\
\hline \multirow[t]{2}{*}{$\mathrm{AF}$} & $\mathrm{CCP}$ & $-0,43506^{*}$ & $-0,49575^{\star}$ & $-0,39904^{*}$ & 0,144155 \\
\hline & $\mathrm{p}$ & 0,005023 & 0,001142 & 0,010753 & 0,374836 \\
\hline \multirow[t]{2}{*}{ D } & $\mathrm{CCP}$ & $-0,26127$ & $-0,26848$ & $-0,61365^{* *}$ & $-0,13988$ \\
\hline & $p$ & 0,103431 & 0,093922 & $2,55 \mathrm{E}-05$ & 0,389324 \\
\hline \multirow[t]{2}{*}{ EGS } & CCP & $-0,16188$ & $-0,34059^{*}$ & $-0,39592^{*}$ & 0,010764 \\
\hline & $\mathrm{p}$ & 0,318287 & 0,031511 & 0,011442 & 0,947441 \\
\hline \multirow[t]{2}{*}{ V } & CCP & $-0,10965$ & $-0,13133$ & $-0,43143^{*}$ & $-0,07886$ \\
\hline & $p$ & 0,500615 & 0,419227 & 0,005443 & 0,628583 \\
\hline \multirow[t]{2}{*}{ AS } & CCP & $-0,31539^{*}$ & $-0,50511^{* *}$ & $-0,60549$ ** & $-0,262$ \\
\hline & $\mathrm{p}$ & 0,047441 & 0,000887 & $3,49 \mathrm{E}-05$ & 0,102436 \\
\hline \multirow[t]{2}{*}{$\mathrm{AE}$} & CCP & 0,176457 & $-0,22768$ & $-0,36246^{*}$ & $-0,18499$ \\
\hline & $p$ & 0,276068 & 0,157663 & 0,021533 & 0,253123 \\
\hline \multirow[t]{2}{*}{ SM } & CCP & 0,040225 & $-0,20958$ & $-0,52895^{* *}$ & $-0,32746^{*}$ \\
\hline & $\mathrm{p}$ & 0,805342 & 0,194304 & 0,00045 & 0,039151 \\
\hline \multirow[t]{2}{*}{ CF - final } & CCP & $0,414976^{*}$ & 0,10538 & $-0,18969$ & $-0,45854^{*}$ \\
\hline & $\mathrm{p}$ & 0,007755 & 0,517533 & 0,241063 & 0,002923 \\
\hline \multirow[t]{2}{*}{ CM - final } & $\mathrm{CCP}$ & $-0,10491$ & 0,273941 & $0,404663^{*}$ & 0,120267 \\
\hline & p & 0,519422 & 0,087172 & 0,009598 & 0,459784 \\
\hline
\end{tabular}

* $p<0,05 \quad$ ** $p<0,01$

CF: capacidade funcional; AF: aspectos físicos; D: dor; EGS: estado geral de saúde; V: vitalidade; AS: aspectos sociais; AE: aspectos emocionais; SM: saúde mental; CF-final: componente físico final; CM-final: componente mental final. 


\section{DISCUSSÃO}

A qualidade de vida é um conceito multidimensional que abrange aspectos físicos, sociais e emocionais. Neste estudo, foram analisados os dados relativos à saúde geral e à qualidade de vida de um grupo de indivíduos com osteoporose.

Os valores obtidos no OPAQ, neste estudo, são concordantes com os dados publicados em outros estudos ${ }^{(15,16,17)}$.

A qualidade de vida avaliada pelo SF-36 mostrou-se diminuída em relação aos valores encontrados na população geral brasileira em todos os domínios. Comparados aos portadores de outra doença reumática, observa-se que a qualidade de vida é diminuída nos domínios capacidade funcional, aspetos físicos, vitalidade e aspectos emocionais ${ }^{(29)}$.

A análise fatorial agrupou em um mesmo fator, o autocuidado, a independência, trabalho doméstico e mobilidade. $\mathrm{O}$ estudo sugere que o autocuidado e a independência estejam diretamente ligados, e que para se poder realizar as atividades de vida diária (AVD) é necessário que o indivíduo também tenha boa mobilidade para executar seu trabalho doméstico do dia-a-dia. Outros autores utilizaram a definição de dependência quando o indivíduo necessita de ajuda até para as AVD, levando a uma diminuição de função ${ }^{(30)}$.

Outro grupo de componentes incluiu flexibilidade, capacidade para realizar transferências e sono. É coerente que uma pessoa com boa flexibilidade consiga realizar mais facilmente as mudanças de postura. Mas é curioso que o sono esteja mais relacionado com flexibilidade e transferência do que com humor e dor. Porém, numa população com osteoporose, a capacidade de mudança de decúbito parece ser um fator determinante para a qualidade do sono. Essa foi uma observação muito interessante e merece que outros trabalhos sejam desenhados para responder especificamente sobre movimentações necessárias nas atividades, inclusive durante o sono, pois não existem trabalhos relacionando sono e osteoporose.

O terceiro fator agrupou os componentes de fadiga, dor e humor. Este dado é concordante com o conceito de que o distúrbio do humor interfere com o limiar de dor. Ainda podemos levantar a hipótese de que estas pacientes com dor e com distúrbio do humor tivessem co-morbidades, como a fibromialgia, uma doença muito comum e com prevalência aumentada na população idosa ${ }^{(31)}$. Segundo Leveille et $a^{(32)}$ a dor musculoesquelética difusa é um fator de risco para quedas em mulheres idosas.

\section{REFERÊNCIAS}

1. World Health Organization. Assessment of fracture risk and its application to screening for posmenopausal osteoporosis: report of a WHO study group. Technical Report Series, n. 843, 1994.
O quarto fator incluiu medo de cair, atividades sociais, apoio familiar e tensão. As pacientes com medo de quedas são as que apresentam mais tensão, preocupação e ansiedade. É possível que esta relação seja bidirecional. Portanto, quem tem mais medo de cair fica mais tenso e quem é mais tenso provavelmente tem mais medo de cair. Todos esses fatores podem interferir no relacionamento familiar, principalmente a perda de independência. Assim, essa doença implica em menor auto-estima, isolamento social e comprometimento psicológico $^{(33)}$.

Cada vez mais reclusas das atividades sociais, as pacientes necessitam muito mais do suporte familiar. Por outro lado, o suporte familiar deve ser fundamental para atenuar a tensão e o medo de cair. Um trabalho com dados semelhantes verificou que intervenções são necessárias num grupo de idosos para reduzir o medo de quedas e melhorar a função social e mobilidade dos indivíduos ${ }^{(34)}$. O isolamento social traz como conseqüência alterações no comportamento psicossocial, como medo, ansiedade e depressão ${ }^{(2)}$.

A diminuição da função em mulheres idosas é devido à combinação de vários fatores como redução dos hábitos de vida saudáveis, obesidade, inatividade física, entre outros ${ }^{(35)}$. Os graus de sofrimento e o desamparo, a redução da capacidade cardíaca e pulmonar, medo de quedas, perda da auto-estima interferem no desempenho dos pacientes na vida profissional, familiar e social ${ }^{(33)}$. Nosso estudo mostrou agrupamento entre desempenho no trabalho e auto-imagem.

Finalmente, houve correlação entre os domínios e componentes finais do SF-36 e os domínios do OPAQ. Os coeficientes de correlação mais altos foram entre o OPAQ Sintomas e alguns domínios do SF-36. O OPAQ Psicológico apresentou correlação estatisticamente significativa com três domínios do SF-36: aspectos físicos, estado geral de saúde e aspectos sociais. O OPAQ Físico se correlacionou melhor com os aspectos físicos, a capacidade funcional e o componente físico final do SF-36. Estes resultados claramente evidenciaram uma concordância entre estes dois instrumentos.

Este estudo permitiu concluir que o OPAQ, um instrumento de avaliação específico para osteoporose, tem capacidade para avaliar aspectos específicos e gerais da qualidade de vida nesta população, sendo concordante com o SF-36.

Declaramos a inexistência de conflitos de interesse.

2. Galsworthy TD, Wilson PL: Osteoporosis - it steals more than bone. Am J Nurs 96: 27-33, 1996.

3. Martin AR, Sornay-Rendu E, Chandler JM, Duboeuf F, Girman CJ, Delmas PD: The impact of osteoporosis on quality-of-life 
- the OFELY cohort. Bone 31: 32-6, 2002.

4. Ioannidis G, Gordon M, Adachi JD: Quality of life in osteoporosis. Nurs Clin North Am 36: 481-9, 2001

5. Adachi JD, Loannidis G, Berger C, et al: The influence of osteoporotic fractures on health-related quality of life in community-dwelling men and women across Canada. Osteoporos Int 12: 903-8, 2001

6. Gold DT: Osteoporosis and quality of life psychosocial outcomes and interventions for individual patients. Clin Geriatr Med 19: 271-80, 2003.

7. Randell AG, Nguyen TV, Bhalerao N, Silverman SL, Sambrook PN, Eisman JA: Deterioration in quality of life following hip fracture: a prospective study. Osteoporos Int 11: 460-6, 2000.

8. Burckhardt CS, Clark SR, Bennett RM: Fibromyalgia and quality of life - a comparative analysis. J Rheumatol 20: 475-9, 1993.

9. Fries JF, Spitz PW, Kraines RG, Holman HR: Measurement of patient outcome in arthritis. Arthritis Rheum 23: 137-45, 1980 .

10. Milligan SE, Hom DL, Ballou SP, et al: An assessment of the health assessment questionnaire functional ability index among women with systemic lupus erythematosus. J Rheumatol 20: 972-6, 1993.

11. Lydick E, Zimmerman SI, Yawn B, et al: Development and validation of a discriminative quality of life questionnaire for osteoporosis - the OPTQoL. J Bone Miner Res 12: 456-63, 1997.

12. Lydick E, Martin A, Yawn B: Impact of fears on quality of life in patients with a silent disease - osteoporosis. Clin Ther 18: 1307-15, 1996

13. Tosteson AN, Hammond CS: Quality-of-life assessment in osteoporosis: health-status and preference-based measures. Pharmacoeconomic 20: 289-303, 2002

14. Chandler JM, Martin AR, Girman C et al: Reliability of an osteoporosis-targeted quality of life survey instrument for use in the community - OPTQoL. Osteoporos Int 8: 127-35, 1998.

15. Silverman SL, Mason J, Greenwald M: The osteoporosis assessment questionnaire (OPAQ) - A reliable and valid self instrument measure of quality of life in osteoporosis. J Bone Min Res 8 (suppl 1): abstract 343, 1993

16. Randell AG, Bhalerao N, Nguyen TV, Sambrook PN, Eisman JA, Silverman SL: Quality of life in osteoporosis - Reliability, consistency, and validity of the osteoporosis assessment questionnaire. J Rheumatol 25: 1171-9, 1998

17. Cantarelli FB, Simões MFJ, Oliveira LM, Ferraz MB, Szejnfeld VL: Qualidade de vida em pacientes com fraturas por osteoporose - Adaptação cultural, reprodutibilidade e validação do Osteoporosis Assessment Questionnaire (OPAQ). Rev Bras Reumatol 39: 9-18, 1999.

18. Badia X, Prieto L, Roset M, Díez-Pérez A, Herdman M: Development of a short osteoporosis quality of life questionnaire by equating items from two existing instruments. J Clin Epidemiol 55: 32-40, 2002.

19. Cook DJ, Guyatt GH, Adachi JD, et al: Development and validation of the mini-osteoporosis quality of life questionnaire (OQLQ) in osteoporotic women with back pain due to vertebral fractures - Osteoporosis quality of life study group. Osteoporos Int 10: 207-13, 1999.

20. Lips P, Cooper C, Agnusdei D, et al: Quality of life in patients with vertebral fractures - Validation of the quality of life questionnaire of the European Foundation for Osteoporosis (QUALEFFO). Working Party for Quality of Life of the European Foundation for Osteoporosis. Osteoporos Int 10: 150-60, 1999.

21. Marquis P, Cialdella P, De la Loge C: Development and validation of a specific quality of life module in post-menopausal women with osteoporosis - the QUALIOST. Qual Life Res 10: 555-66, 2001.

22. Ailinger RL, Lasus H, Braun MA: Revision of the facts on osteoporosis quiz. Nurs Res 52: 198-201, 2003.

23. Coelho R, Silva C, Maia A, Prata J, Barros H: Bone mineral density and depression: a community study in women. J Psychosom Res 46: 29-35, 1999.

24. Ware JE, Sherbourne CD: The MOS 36 items short-form health survery (SF-36) - 1. Conceptual framework and item selection. Med Care 30: 473-83, 1992.

25. Ware JE, Snow KK, Kosinski M: SF-36 Health Survery - Manual and interpretation guide. Boston: Health Institute. New Eng Med Center, 1993.

26. Ciconelli RM, Ferraz MB, Santos W, Meinão I, Quaresma MR: Tradução para a língua portuguesa e validação do questionário genérico de avaliação de qualidade de vida SF-36 (Brasil SF-36). Rev Bras Reumatol 39: 143-50, 1999.

27. Díaz MN, López JBD, Rebollar AR, Alonso CG, Corte CD, Andía JC: Efecto de la fractura vertebral sobre la calidad de vida relacionada con la salud en población asturiana mayor de 54 años. Med Clin (Barc) 116: 533-5, 2001.

28. Kanis JA: Assessment of fracture risk and its application to screening for postmenopausal osteoporosis - Synopsis of a WHO Report. WHO Study Group. Osteoporos Int 4: 368-81, 1994.

29. Senna ER, De Barros AL, Silva EO, et al: Prevalence of rheumatic diseases in Brazil: a study using the COPCORD approach. J Rheumatol 31: 594-7, 2004.

30. Jagger C, Spiers NA, Clarke M: Factors associated with decline in function, institutionalization and mortality of elderly people. Age Ageing 22: 190-7, 1993

31. Gowin KM: Diffuse pain syndromes in the elderly. Rheum Dis Clin North Am 26: 673-82, 2000.

32. Leveille SG, Bean J, Bandeen-Roche K, Jones R, Hochberg M, Guralnik JM: Musculoskeletal pain and risk for falls in older disabled women living in the community. J Am Geriatr Soc 50: 671-8, 2002.

33. Gold DT: The clinical impact of vertebral fractures - quality of life in woman with osteoporosis. Bone (supl 3): 185-9, 1996.

34. Tennstedt S, Howland J, Lachman M, Peterson E, Kasten L, Jette A: A randomized, controlled trial of a group intervention to reduce fear of falling and associated activity restriction in older adults. J Gerontol B Psychol Sci Soc 53: 384-92, 1998.

35. Ensrud KE, Nevitt MC, Yunis C, et al: Correlates of impaired function in older women. J Am Geriatr Soc 42: 481-9, 1994. 